\title{
Estudiantes universitarios en riesgo de exclusión social: una aproximación a una realidad social oculta
}

\section{University students at risk of social exclusion: an approach to a hidden social reality}

\author{
Débora García Barrios, Jorge Luis Méndez-Ulrich¹
}

\section{Resumen}

La reciente recesión económica ha ocasionado un gran impacto en las condiciones de vida de la población española. En el presente estudio se realiza una aproximación al efecto de la crisis económica en el acceso a los estudios universitarios, así como a los comportamientos relacionados con la exclusión social que los estudiantes universitarios serían capaces de realizar o ya han realizado, con el único propósito de costearse su educación. Para ello, 96 estudiantes contestaron voluntariamente una encuesta cuyo objetivo era evaluar su situación sociolaboral. detectar situaciones de riesgo de exclusión, y una escala actitudinal en relación a conductas de riesgo social. A pesar de que el 78,5\% de los encuestados trabajaba, un 79,2\% percibía su situación económica como media o baja. Un 77,3\% de encuestados habían realizado a lo largo de sus estudios a conductas de riesgo como trabajar sin contrato, traficar con drogas, o incluso prostituirse. De forma consecuente, los resultados sugieren una asociación entre el nivel económico y riesgo social. por lo que se daría la paradoja de que la universidad constituya una puerta de entrada a la exclusión. Finalmente, se comentan las limitaciones del estudio y se destaca la necesidad de profundizar en el abordaje de este fenómeno.

\section{Palabras clave}

Estudiantes universitarios, universidad, pobreza, exclusión social.

\section{Abstract}

The recent economic recession has had a great impact on the living conditions of the Spanish population. In the present study, an approximation is made to the effect of the economic crisis on the access to university studies, as well as the behaviors related to social exclusion that university students would be able to perform or have already done, for the sole purpose of funding their education. For this, 96 students voluntarily answered a survey whose objective was to evaluate their socio-laboral situation, to detect situations of risk of exclusion, and an attitude scale in relation to social risk behaviors. Although $78.5 \%$ of the respondents had a job, $79.2 \%$ perceived their economic situation as average or low. $77.3 \%$ of respondents had carried out, throughout their studies, risk behaviors such as working without a contract, dealing with drugs, or even prostitution. Consequently, the results suggest an association between

1 Universidad Autónoma de Barcelona: d.garciabarrios@gmail.com, jordi.mendez@ub.edu 
economic level and social risk, and this would lead to the the paradox that access to the university constitutes a gateway to exclusion. Finally, the limitations of the study are discussed, and the need to dive deeper into the approach of this phenomenon is highlighted.

\section{Key words}

Higher education, university, poverty, social exclusion.

\section{Marco teórico}

\subsection{Introducción}

El inicio formal de la crisis económica en la que nos hallamos actualmente inmersos se asocia con el denominado «estallido de la burbuja inmobiliaria», y la crisis financiera mundial de 2008. Este escenario adquirió una nueva dimensión a partir de la quiebra de la multinacional financiera Lebman Brothers, que tuvo un importantísimo impacto económico a nivel mundial. El Estado Español no quedó al margen de este fenómeno (Alonso y Furió, 2010), y sus cotas de desempleo experimentaron un incremento del 11,94\% entre 2008. cuando la tasa de parados era del 13,79\%, según datos de la Unión General de Trabajadores (UGT) y 2014, cuando se alcanzó el 25,73\%. Cataluña, siguiendo esta tendencia, experimentó un crecimiento de las desigualdades sociales, especialmente en los sectores sociales más vulnerables, como los jóvenes, entre los cuales se encuentra gran parte de los estudiantes universitarios.

Fruto de las medidas en materia económica y laboral impulsadas por el gobierno central y el autonómico, se produjeron recortes salariales, con la consecuente reducción de la capacidad adquisitiva de la población. Estos recortes también afectaron a los presupuestos destinados a asuntos sociales como la sanidad, la educación, etc., lo que ha ido desgastando el conocido como «Estado de Bienestar», en el que se basa la propia definición del Estado expresada en el artículo 1.1 del Título Preliminar de la Constitución Española de 1978, que dice textualmente:

\section{«España se constituye en un Estado Social y democrático de Derecho, que propugna como valores} superiores de su ordenamiento jurídico a la libertad, la justicia, la igualdad y el pluralismo político».

Las condiciones de trabajo se precarizaron a causa de la Reforma Laboral de 2012, como se constata observando la tasa de desempleo del último trimestre de 2013, que alcanzó el 26,03\%, Según la UGT (2014), el número de menores de 35 años desempleados ha ascendido hasta el $47 \%$ en los últimos años, lo que comporta un incremento del $8 \%$ respecto a los registros anteriores a 2008. Para los sindicatos, esta reforma significó «trabajar más y peor por menos», y de acuerdo con el informe de 2014 de la Secretaria de acción sindical de la UGT, esta norma condujo a un incremento de los contratos precarios, ya fueran temporales, de formación o de prácticas (incluso aquellos no ajustados al perfil formativo de los empleados). Además, se redujo la contratación de personas con diversidad funcional, y se incrementaron los despidos colectivos, dado su abaratamiento. La entrada en vigor de esta reforma redundó en el beneficio de las empresas mediante la modificación de las condiciones de trabajo, 
como ilustra el artículo 41.1 del Real Decreto de Ley 3/2012, febrero 2012, de medidas urgentes de reforma del mercado laboral (convertida en Ley 3/2012, de 6 de julio de 2012), que dice textualmente: «La dirección de la empresa podrá acordar modificaciones sustanciales de las condiciones de trabajo cuando existan probadas razones económicas, técnicas, organizativas o de producción. Se consideran tales las que estén relacionadas con la competitividad, productividad u organización técnica o del trabajo en la empresa». Entre las condiciones de trabajo que esta ley permite flexibilizar a criterio de las compañías se hallan. entre otras: aquellas que afectan a horarios y jornadas de trabajo, el régimen de trabajos a turno o el sistema de remuneraciones, lo que incrementó la indefensión de los trabajadores, y fomentó una mayor arbitrariedad por parte de las empresas a la hora de fijar estas condiciones.

El impacto social de estas reformas y recortes se ven también reflejados en trabajos como los de Laparra et al., (2010) o Lorenzo et al., (2014), y en las encuestas de instituciones como el Institut a de Catalunya (2012) o el Instituto Nacional de Estadística (2014), que muestran no solo una degradación de las condiciones de trabajo en el sector privado, sino también en el público, viéndose afectadas las dotaciones destinadas a las prestaciones sociales de las que amplios sectores sociales dependen, incluidos los recursos para el sistema público universitario.

\subsection{Crisis, pobreza y exclusión}

Los datos correspondientes a 2014 del EUROSTAT muestran como el 29,2\% de la población española se encuentra en riesgo de exclusión social, siendo este dato 5,2 puntos porcentuales superior la media de la Unión Europea, que es del 24,4\%. Este deterioro en las condiciones de vida tiene su reflejo en el incremento del desempleo y de las desigualdades sociales, así como en el aumento del número de desahucios, de la demanda de servicios de asistencia social, y del número de personas que tuvieron que volver al domicilio familiar por no disponer de ingresos para vivir de forma independiente.

La Organización de las Naciones Unidas (1995) define la pobreza como «La condición caracterizada por una privatización severa de necesidades humanas básicas, incluyendo alimentos, agua potable, instalaciones sanitarias, salud, vivienda educación e información. La pobreza depende no solo de ingresos monetarios sino también del acceso a servicios». Por otra parte, Subirats (2004:2) afirma que la pobreza es un complejo de realidades que abarcan, más allá de la desigualdad económica, aspectos relacionados con la precariedad laboral, los déficits de formación, el difícil acceso a una vivienda digna, las frágiles condiciones de salud y la escasez de redes sociales y familiares, entre otros. La primera de estas definiciones procede de un organismo oficial, por lo que se trata de una operacionalización del fenómeno de la pobreza; mientras que la segunda representa una aproximación academicista a esta realidad. No obstante, ambas definiciones enfatizan que la pobreza se define básicamente por la ausencia de recursos materiales, aunque esta situación no represente un riesgo para la supervivencia. Desde este punto de vista, en la actualidad la pobreza se manifiesta frecuentemente y mediante múltiples formas como el paro de larga duración, la cronificación en la dependencia de ayudas sociales e incluso el impago y corte de los suministros básicos, entre otras muchas.

Por otra parte, se entiende por exclusión social «el proceso social de separación de un individuo o grupo respecto a las posibilidades laborales, económicas, políticas y culturales a las que otros sí tienen acceso y disfrutan» (Gil, 2002:21). Este proceso lo pueden desatar multitud de situaciones como el desempleo, disponer de una vivienda deficiente, las dificultades en el acceso a la educación, o en general, cualquier limitación en la participación social (Bel Adell, 2002:3). Otros autores como Hernández Pedreño (2010) subrayan el hecho de que las propias instituciones europeas huyan de los criterios exclusivamente economicistas a 
la hora de definir qué es la exclusión social, destacando su carácter multidimensional y procesual. Por tanto, existe en la sociedad una cierta «división invisible» entre aquellos ciudadanos que pueden hacer frente a las consecuencias de la crisis económica y vivir con una adecuada calidad de vida, y aquellos que se acercan mucho a la exclusión social, cuando no se ubican en ella. Subirats (2004) también afirma que la exclusión social es un proceso dinámico que se produce como «[...] resultado de una cadena de acontecimientos reforzados o impulsados por las desigualdades y determinaciones estructurales del sistema económico sociali. Este autor señala los principales ejes de desigualdad social (el género, la edad y la procedencia), y apunta a los tres pilares básicos de la inclusión, que pueden verse seriamente afectados en situaciones crisis, y que son: 1) la participación en la producción y en la creación de valores, 2) la adscripción cultural y ciudadana, y 3) la adscripción política y conexión con redes de apoyo social. A pesar de que estas definiciones fueron postuladas a principios del pasado siglo, y de que con poca frecuencia se asocian al conocido como primer mundo, se ajustan a la realidad de muchas personas en nuestro entorno inmediato.

\subsection{El impacto de la crisis en el acceso a la universidad}

En este contexto de crisis social y económica, los jóvenes no solo han experimentado graves dificultades en cuanto a su inserción laboral (Agencia para la Calidad del Sistema Universitario de Cataluña, 2014), siendo actualmente la tasa de paro juvenil superior al 50\% en el conjunto de nuestro país, sino estas dificultades en el acceso al mercado laboral son superiores en aquellos titulados universitarios de clases sociales más desfavorecidas (Navarro, 2014). Estas dificultades para los jóvenes de clases obreras, también existen en cuanto a acceder y/o mantenerse en el sistema universitario, debido en gran medida al progresivo incremento que los precios y tasas universitarias han experimentado en paralelo al empobrecimiento de la población (Langa Rosado y Río Ruiz, 2013; Hernández Armenteros y Pérez García, 2013). El Informe ejecutivo sobre condiciones vida y participación de los estudiantes de la Universitat de Barcelona (Observatori de l'Estudiant de la UB, 2016) señala como, en el momento actual, a pesar que cursar estudios universitarios fomenta la movilidad social, persisten las diferencias en el acceso a la universidad en función de la extracción social de los padres de los y las estudiantes. En este sentido, a partir de la aplicación del Plan de Bolonia, a partir del curso 2012-2013 el precio del crédito universitario experimentó un aumento significativo, siendo Cataluña la Comunidad Autónoma donde se experimentó el mayor incremento $(66,6 \%)$ respecto al curso anterior, en la que más resulta más caro estudiar en la universidad pública (Escardíbul et al., 2013; Andreu y Pérez, 2014). Las políticas en materia educativa llevadas a cabo durante los últimos años han tendido al beneficio del sector privado y al perjuicio del público, como se pone de manifiesto con leyes como la Ley Orgánica para la Mejora de la Calidad Educativa de 2013, o el Real Decreto 609/2013, 2 de agosto, por el que se establecen umbrales de renta y patrimonio familiary las cuantías de las becas y ayudas al estudio para el curso 2013-2014, y se modifica particularmente el real decreto 1721/2007, de 21 de diciembre, por el que se establece el régimen de las becas y ayudas al estudio personalizado. Con esta norma se reducen o suprimen ayudas como aquellas destinadas a desplazamiento, ayudas residenciales, material para el estudio, realización del proyecto final de carrera, ayudas por rendimiento académico o complementos para cursar másteres.

Además, esta reforma dificulta el acceso a las ayudas, al incorporar un componente variable que considera para su concesión no solo la nota media y la renta familiar del solicitante, sino también el número de becados; al mismo tiempo que se endurecieron los requisitos de rendimiento académico para acceder a una beca, ya que se exige una mayor nota en las pruebas de acceso a la universidad, 
teniéndose que aprobar durante los cursos posteriores un mínimo del $85 \%$ de créditos matriculados en el caso de las enseñanzas técnicas, y del 100\% en el resto. Incluso se contempla el supuesto de que se deba reembolsar el importe íntegro de la beca en caso de no superar el 50\% de créditos (Río Ruiz, 2014).

Como derivada de esta situación, y de las barreras con la que los y las jóvenes han de enfrentarse para acceder a la universidad (como la falta de trabajo o la clase social de sus padres), cabe preguntarse hasta qué punto los propios estudiantes universitarios podrían estar exponiéndose a situaciones de riesgo de exclusión social como la prostitución, el tráfico de drogas, el trabajo precario, etc., con el único propósito de poder costearse sus estudios, lo que supondría una situación de emergencia para el sistema universitario, y exigiría una rápida y contundente respuesta institucional. Este estudio, que representó un Trabajo Final de Grado (TFG) de Educación Social por la Universitat de Barcelona se centra en una muestra de estudiantes de esta materia durante el curso 2013-2014, en el que podría estar produciéndose la paradoja de que estos futuros profesionales que han de combatir la exclusión se estarían exponiendo a ella, precisamente para poder alcanzar su capacitación profesional.

\section{Método}

Se optó por una metodología de carácter descriptivo ya que el objetivo del estudio era aproximarse a un fenómeno aún inexplorado: la situación socioeconómica y laboral de los y las estudiantes de último curso del Grado de Educación Social de la Universitat de Barcelona; y hasta qué punto esta podría relacionarse con la realización de comportamientos vinculados a la exclusión social, para poder costearse sus estudios, y con la actitud respecto a realizarlos en caso de necesidad. Por otra parte, se optó por la metodología cuantitativa debido a: (1) el mencionado carácter descriptivo y exploratorio del estudio (2) la necesidad de acceder a una muestra de estudiantes lo más numerosa posible, (3) la dificultad que supondría procesar y analizar una cantidad elevada de información cualitativa, y (4) la confidencialidad que garantiza el empleo del método de encuesta, el cual facilita que las respuestas a preguntas potencialmente comprometedores sean lo más sinceras posibles, y se vean lo menos afectadas por un efecto de «deseabilidad social».

\subsection{Preguntas de investigación}

Debido al carácter exploratorio del estudio, no se establecieron hipótesis específicas, sino que se trabajó a partir de las siguientes preguntas de investigación:

- ¿Cuál es el perfil socioeconómico y laboral de los y las estudiantes de último curso del Grado de Educación Social de la Universitat de Barcelona?

- ¿Hay estudiantes de Educación Social que hayan realizado comportamientos de riesgo de exclusión social con el único propósito de costearse sus estudios?

- ¿Cuál es la prevalencia de estos comportamientos entre los y las estudiantes de $4 .^{\circ}$ curso del Grado en Educación Social en la Universitat de Barcelona?

- ¿Hasta qué punto existe una disposición por parte de estos estudiantes a realizar voluntariamente comportamiento de riesgo para poder costearse la carrera?

\subsection{Participantes}

En el estudio participaron 96 estudiantes de $4 .^{\circ}$ curso del Grado en Educación Social de la Universitat de Barcelona (77 mujeres y 19 hombres), del total de 178 estudiantes matriculados de la asignatura 
Trabajo Final de Grado. Se trató de una muestra accidental (ya que participaron aquellos estudiantes que voluntariamente accedieron a contestar el cuestionario), pero relativamente representativa del conjunto de estudiantes de último curso de esta titulación, ya que: 1) representa un 53,4\% de estudiantes de cuarto curso, 2) estos estudiantes, al tener un recorrido dentro de la institución universitaria de al menos 3 años, han experimentado en primera persona el incremento de los precios y las modificaciones en la política de becas iniciados en el curso 2012-2013. El 24\% de la muestra encuestada no trabajaba en el momento del estudio. Ya que el objetivo principal de este trabajo era identificar la hipotética paradoja que supone el hecho de que los futuros profesionales de la Educación Social se vean inmersos en situaciones de exclusión para alcanzar su capacitación profesional a pesar del endurecimiento del contexto y las medidas políticas adoptadas, el único criterio para participar en la investigación fue estar en el último año del grado en el momento del estudio (curso 2013-2014), sin incluir ningún otro tipo de variables de exclusión.

\subsection{Elaboración del instrumento de recogida de información}

Para realizar el trabajo de campo se creó un instrumento a partir de la adaptación del cuestionario empleado por el Observatorio del Estudiante de la UB para realizar el registro de la situación socioeconómica de sus estudiantes. Este cuestionario fue ampliado mediante la inclusión de una serie de ítems relativos a las dimensiones que se incluyeron en el cuestionario final fueron:

- Una primera dimensión descriptiva relativa a la información sociodemográfica de los participantes.

- Otra relativa a la situación socioeconómica y laboral, y a la percepción del impacto de las políticas universitarias en una escala de tipo Likert, con 4 opciones de respuesta: «nada», «un poco», «bastante» $\mathrm{y}$ «mucho».

- Una última dimensión mediante la cual se evaluaba el grado de exposición a comportamientos de riesgo social y su actitud respecto a llevarlos a cabo en el futuro, empleando una escala actitudinal con un rango de 0 a 10 puntos.

\subsection{Procedimiento}

En primer lugar, se contactó mediante un email con los docentes del TFG, solicitando su autorización para realizar la recogida de datos durante los 10 primeros minutos de una de sus sesiones de clase. En el momento de la obtención de información, la encuestadora $(n=1)$ indicaba en líneas generales el propósito del estudio, y para evitar un posible efecto de deseabilidad social, informaba de que los datos obtenidos serían tratados de acuerdo a la Ley Orgánica 15/1999, de 13 de diciembre, de Protección de Datos de Carácter Personal, por lo que quedaba totalmente garantizada la confidencialidad y el anonimato de las respuestas. También se indicó a los participantes que, ante cualquier duda, podían solicitarle cuantas aclaraciones fuesen necesarias. Finalmente, se informó de que no había respuestas correctas ni incorrectas, y de que se debía contestar las preguntas con la mayor rapidez posible. El tiempo medio que demoraba la aplicación del cuestionario fue de aproximadamente diez minutos.

\subsection{Plan de análisis de datos}

Los datos de las encuestas fueron introducidos en una matriz de datos construida mediante la plataforma Google Forms. Posteriormente se generó la matriz final de datos mediante el software IBM SPSS (versión 21), para Windows. Los análisis estadísticos consistieron en el cálculo de medias con 
sus correspondientes desviaciones estándar y en el cálculo de frecuencias y porcentajes, tanto para la descripción sociodemográfica de la muestra como para la evaluación de la prevalencia de comportamientos de riesgo social ya realizados por los participantes en el estudio. Finalmente, para la comparación de medias obtenidas en la escala actitudinal en función del género se emplearon pruebas de análisis de la varianza univariantes (ONEWAY Anova).

\section{Resultados}

\subsection{Características sociodemográficas y sociolaborales de la muestra}

La muestra estuvo compuesta por un $80,2 \%$ de mujeres y un $19,8 \%$ de hombres. La media de edad fue de 26,84 años (D.T. $=6,29)$, siendo de 26,09 (D.T. $=5,33$ ) en el caso de las mujeres y de 29,84 (D.T.=6,29) en el de los hombres. Respecto a la percepción de la propia situación económica, el 39,6\% de los participantes consideraron su renta como baja, el 39,6\% como media-baja, el 18,8\% como media, y solo un $2 \%$ la consideran media-alta o como alta. En cuanto a su situación sociolaboral, los participantes mayoritariamente vivían con sus padres (44,8\%), seguidos por un $24 \%$ que convivían en pareja, y el $(16,7 \%)$ que compartían piso. Respecto a su situación económica, en la mayoría de casos la principal fuente de ingresos provenía de las rentas del trabajo (38,5\%), seguida de ayudas familiares $(21,9 \%)$. A este respecto, el 60,5\% de los estudiantes que percibían apoyo económico de sus familiares, lo recibían de parte de sus padres. En cuanto a la situación laboral de los participantes, un 75,8\% de la muestra trabajaba en el momento del estudio. No obstante, del 24,2\% de estudiantes que no trabajan, el 60,9\% no estaban inscritos en el registro de desempleados, En cuanto a la dedicación, un 59,5\% de los participantes trabajaban a jornada parcial, un 21,6\% trabajaba a jornada completa, y un 14,9\% lo hacía de forma intermitente. Un 4,1\% informó de disponer de dos trabajos (uno a tiempo parcial y otro esporádico; o dedicación completa en uno y parcial en el otro). No obstante, representa un dato relativamente positivo el hecho de que el tipo de contrato más habitual entre los participantes en el estudio fuera el de carácter indefinido $(35,5 \%)$, seguido del eventual (32,9\%). En cuanto a las becas obtenidas por los estudiantes encuestados a lo largo de los dos años previos al estudio, un 39,6\% no había recibido ningún tipo de ayuda (ni Beca de Equidad ni Beca General).

\subsection{Comportamientos de riesgo}

El Gráfico I resume aquellos comportamientos relacionados con el riesgo social que los participantes informaron haber realizado a lo largo de su paso por la universidad, con el único propósito de poder costearse los estudios. Como puede observarse, el comportamiento de riesgo social más prevalente en nuestro estudio fue el de haber trabajado en el pasado sin contrato (35,8\%). De hecho, un 21,1\% de participantes afirmó que lo hacía en el momento de contestar la encuesta, por lo que el total de estudiantes que en algún momento ha trabajado sin contrato ascendió al 56,9\%. Además, el 2,1 \% en algún momento había cobrado por sus apuntes o realizado trabajos para otras personas: y un $1 \%$ lo hacía en el momento del estudio, por lo que el porcentaje total de este «fraude académico» (en tanto que se altera el rendimiento académico de terceras personas mediante una cierta suplantación de la identidad por parte del estudiante que realiza estos trabajos a cambio de dinero) era del 3,1\%. Un 5,2\% de los estudiantes había participado en algún momento en ensayos clínicos, y un 2,1\% había donado semen u óvulos para obtener el dinero necesario para poder financiarse la carrera. Finalmente, un 6,3\% manifestó que había traficado con drogas, e incluso un participante declaró haberse prostituido para poder mantener sus estudios universitarios. 


\section{Gráfico I. Frecuencia de realización de conductas de riesgo}

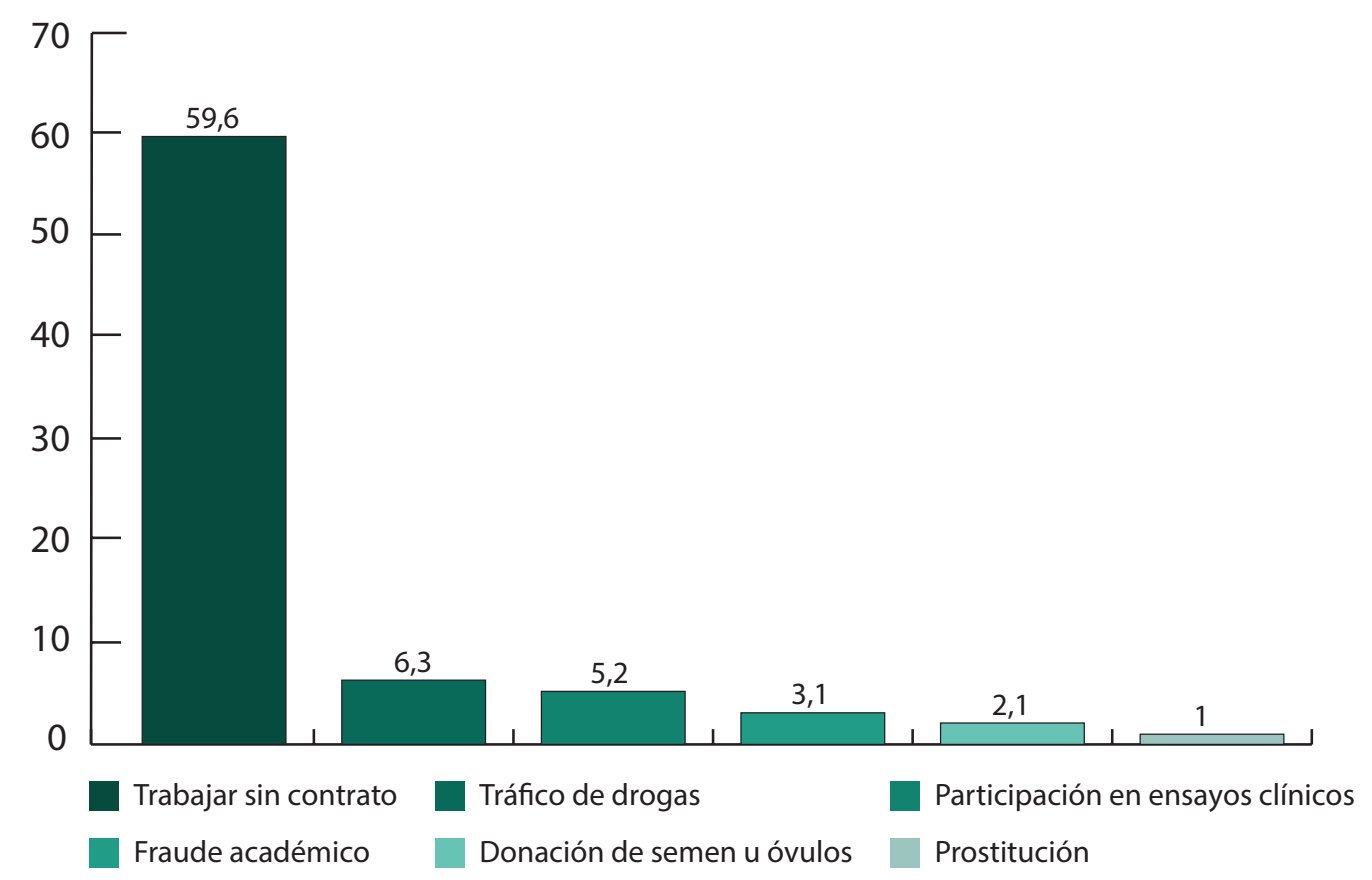

\subsection{Actitudes respecto a los comportamientos de riesgo.}

Tal como se resume en el Gráfico II, el 97,9\% de participantes mostraron en la escala actitudinal en mayor o menor medida, su disposición a trabajar sin contrato, y un $87,5 \%$ se valoraba la posibilidad de comerciar con sus apuntes y trabajos para ganar dinero. El 72,6\% de encuestados se manifestaron dispuestos a participar en ensayos clínicos o a donar óvulos o esperma $(73,4 \%)$ a cambio de dinero. Finalmente, el 66,7\% se manifestaba en cierta medida dispuestos a traficar con drogas, e incluso un relevante $28,1 \%$ no descartaba completamente la posibilidad de prostituirse para poder financiarse la carrera.

\section{Gráfico II. Tasa de estudiantes dispuestos a realizar comportamientos de riesgo para pagar sus estudios}

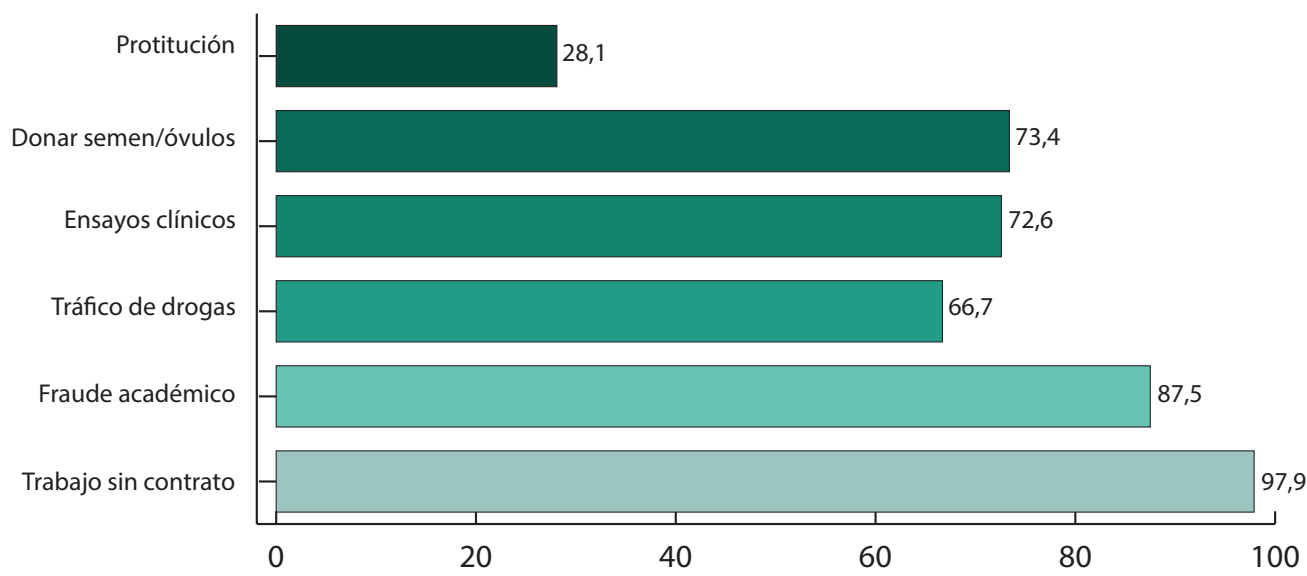


Por su parte, la Tabla I expresa la puntuación media en la escala actitudinal (rango 0-10) de los estudiantes que mostraron algún tipo de intención de realizar los comportamientos de riesgo evaluados y reflejan el nivel de predisposición a llevarlas a cabo en caso de ser preciso para financiarse los estudios.

Tabla I. Medias en la puntuación de la escala actitudinal (disposición a realizar conductas de riesgo)

\begin{tabular}{|c|c|c|c|c|c|c|}
\hline & $\begin{array}{c}\text { Media } \\
\text { en la escala }\end{array}$ & Renta baja & $\begin{array}{l}\text { Renta media- } \\
\text { baja }\end{array}$ & Renta media & Hombres & Mujeres \\
\hline Trabajar sin contrato & 8,80 & 9,68 & 8,50 & 7,31 & 7,42 & 8,91 \\
\hline Fraude académico & 7,12 & 7,76 & 6,55 & 6,67 & 4,68 & 6,61 \\
\hline Tráfico de drogas & 6,38 & 6,86 & 6,13 & 5,55 & 4,10 & 4,30 \\
\hline Ensayos clínicos & 5,41 & 5,71 & 5,26 & 4,22 & 3,89 & 4,01 \\
\hline Donación de semen u óvulos & 6,02 & 6,00 & 5,90 & 6,00 & 6,11 & 4,04 \\
\hline Prostitución & 4,69 & 4,25 & 4,42 & 4,25 & 2,47 & 1,04 \\
\hline
\end{tabular}

Además, se observó cómo estas actitudes eran generalmente mayores en aquellos estudiantes con rentas bajas, por lo que se trata de un colectivo especialmente vulnerable a la exclusión social. Los comportamientos en los que los participantes se mostraron más dispuestos a realizar fueron en este grupo, de mayor a menor: trabajar sin contrato, realizar trabajos universitarios para terceras personas a cambio de dinero, traficar con drogas, donar óvulos o esperma, participar en ensayos clínicos remunerados y, finalmente, prostituirse. En cuanto al género, las mujeres puntuaron de forma estadísticamente significativa por encima de los hombres en su actitud respecto a trabajar sin contrato ( $F=7,650 ; \mathrm{p}=.007)$, y en cuanto a vender sus apuntes o realizar trabajos universitarios pata terceros $(\mathrm{F}=6,815 ; \mathrm{p}=.011)$. Por su parte, los hombres obtuvieron puntuaciones medias superiores a las mujeres (siendo estas diferencias de medias estadísticamente significativas) en cuanto a su actitud en relación a la posibilidad de donar esperma $(F=4,637 ; p=.034)$, y prostituirse $(F=5,751 ; p=.018)$. Finalmente, no se observaron diferencias estadísticamente significativas entre hombres y mujeres en relación a su predisposición a traficar con drogas y a participar en ensayos clínicos.

\section{Discusión}

En primer lugar, en cuanto al impacto de los recortes en las políticas en materia de becas realizados durante los años de recesión económica, éste se ve reflejado en nuestro estudio, dado que un alto porcentaje de participantes que las habían solicitado (prácticamente en 40\%) no había recibido ningún tipo de ayuda al estudio. En lo referente a la situación laboral de nuestros encuestados, a pesar de que la mayoría de ellos declaró disponer de trabajo, la mayor parte de ellos percibía su propia situación económica como media-baja o directamente baja. Esto sugiere que reciben una escasa retribución por su trabajo, y que, en general, desarrollan sus actividades laborales en unas condiciones precarias. La existencia de esta precariedad laboral se ve apoyada por el hecho de que, aunque una gran proporción de encuestados convivía con sus familias, y manifestaba que su principal fuente de ingresos era su propio trabajo, de hecho, dependían de la ayuda económica proporcionada por sus familiares para poder costearse sus estudios universitarios. También en relación a la precariedad en la que desarrollaban su trabajo, los resultados revelaron que la dedicación laboral más habitual era aquella a tiempo parcial (en sintonía con la tendencia general del mercado laboral en nuestro país), e incluso una gran parte disponían de una o más ocupaciones sin un contrato laboral, aunque ciertamente, se observó que el 35,5\% de los participantes que trabajaban en el momento del estudio lo hacían con un contrato indefinido. 
Por tanto, a pesar de que los participantes no descartaban abandonar sus estudios ante su complicada situación socioeconómica, la mayoría la afrontaban alternando estudios y trabajo, o incluso acudiendo a alternativas menos lícitas y relacionadas con la misma exclusión social contra la cual quieren intervenir en su futuro profesional, cómo el tráfico de drogas, entre otras. En cuanto a estos comportamientos relacionados con la exclusión social que los estudiantes manifestaron haber realizado en alguna ocasión para financiarse los estudios universitarios, un elevado número de participantes habían trabajado sin contrato (con la consiguiente vulneración de sus derechos y la exposición a situaciones de vulnerabilidad e indefensión). Además, se registraron casos en todos los comportamientos de riesgo evaluados, incluso en lo referente al tráfico de drogas o la prostitución, lo que constituye una realidad francamente alarmante, e incluso dramática.

También resulta significativo el hecho de que la práctica totalidad de los participantes declaró que, si fuera necesario para pagarse la carrera, estaban dispuestos a realizar alguno de los comportamientos de riesgo evaluados en la escala, aunque en el pasado nunca se lo hubieran planteado. De hecho, se registró un elevado índice de estudiantes dispuestos a trabajar sin contrato y a traficar con drogas, si se diera el caso de que sus estudios dependieran de ello. Respecto a las diferencias de género, las mujeres se mostraron más predispuestas a trabajar sin contrato y a realizar trabajos académicos para terceros, mientras que los hombres mostraron una mayor disposición a ejercer la prostitución y a donar esperma. En cuanto a la participación en ensayos clínicos o traficar con drogas, no se observaron diferencias estadísticamente significativas entre hombres y mujeres.

Además, se observó una posible relación inversa entre la percepción del propio nivel adquisitivo y la realización de conductas de riesgo social, o la disposición de llevarlas a cabo en el futuro. Nuestros resultados sugieren que los estudiantes con menor nivel adquisitivo se pueden ver en ocasiones forzados a aceptar trabajar en condiciones precarias durante sus estudios, cuando no a exponerse a otras situaciones que no se plantearían si no fuera por la necesidad de obtener los recursos mínimos para poder mantenerse en la universidad. Cabe incluso reflexionar sobre hasta qué punto esto podría comprometer su rendimiento académico, dada la interferencia de estas situaciones de riesgo en el correcto seguimiento por parte de estos y estas estudiantes de las actividades académicas. Además, cabría también profundizar en la posibilidad de que, estas situaciones y conductas con las que han entrado en contacto a lo largo de sus estudios se pudieran cronificar, por lo que paradójicamente su paso por la Universidad, en vez de haber actuado como un ascensor social, habría precisamente supuesto su puerta de entrada a la exclusión. En estos casos estaríamos delante de una realidad hasta ahora inexplorada: la de los estudiantes universitarios en situación de riesgo de exclusión social a causa de su propia motivación académica.

Por tanto, en el futuro es preciso realizar estudios más amplios y profundos en relación al impacto de la crisis económica y sus derivadas, no solo en los estudiantes de Educación Social de la Universitat de Barcelona a los que se pudo acceder para este estudio, sino a nivel global del sistema universitario, donde según el tipo de grado y de Comunidad Autónoma, las diferencias en los precios de matrículas son notables (Andreu y Pérez, 2014). Cabe además, emprender un proceso de reflexión dentro de la institución académica respecto a cómo prevenir y detectar estos casos, y especialmente en relación a cómo actuar para revertir estas situaciones una vez son una realidad, para alejar a los estudiantes de la exclusión más o menos latente en la que se puedan hallar inmersos.

No obstante, hay que valorar nuestros resultados con cautela debido a sus limitaciones metodológicas (tamaño de la muestra, sesgo en el tipo de población evaluada, que pertenece a un segmento muy concre- 
to de una determinada institución universitaria, etc.), que han de ser tenidas en cuenta en estos hipotéticos estudios futuros. Ciertamente, nuestra muestra no es representativa de toda la población universitaria y además se ha de considerar el grado de compromiso que podría significar la respuesta a algunas preguntas planteadas en la encuesta podría haber provocado un efecto de «deseabilidad social» que habría sesgado, al menos parcialmente, nuestros resultados. No obstante, este estudio apunta hacia una preocupante realidad, fruto del pasado y del presente contexto económico, así como de las políticas públicas llevadas a cabo para afrontarlo. Esta hipotética realidad exige la atención de las instituciones públicas (especialmente la universitaria), las cuáles han de asumir la responsabilidad de velar por la igualdad de derechos y oportunidades de los jóvenes, no ya solo en cuanto al acceso a la formación universitaria, sino también en la prevención del abandono académico por causas sociales, y de las conductas de riesgo que puedan estar dispuestos y dispuestas a realizar para evitarlo, y sus consecuencias a nivel psicológico, social y moral.

\section{Referencias bibliográficas}

Alonso, Matilde y Furió, Elíes (2010): "La economía española. Del crecimiento a la crisis pasando por la burbuja inmobiliaria". Cabiers de civilisation espagnole contemporaine. De 1808 an temps présent, (6). Doi: $10.4000 /$ ccec.3212.

Elías Andreu, Marina y Daza Pérez, Lidia (2014): "Sistema de becas y equidad participativa en la Universidad". Revista de la Asociación de Sociología de la Educación, 7 (1), 233-251.

Agència Catalana de Qualitat Universitària (2014). Universitat i treball a Catalunya. Informe de la inserció laboral de la població catalana de les universitats catalanes. Disponible online en http://www.aqu.cat/ doc/doc_14857668_1.pdf, consultado el 20 de noviembre de 2016.

Bel Adell, Carmen (2002): “Exclusión social: Origen y características”. Ponencia presentada en Formación específica en Compensación Educativa e Intercultural para Agentes Educativos, 30 de enero, en Murcia. Disponible en: http://enxarxats.intersindical.org/nee/CE_exclusio.pdf.

Constitución Española. (BOE núm. 311, 29 de diciembre de 1978).

EUROSTAT (2015). Statistics on income, social inclusion and living conditions. Disponible en: http:// ec.europa.eu/eurostat $/$ tgm $/$ table.do?tab=table\&init=1\&language $=$ en\&pcode=t2020_50\&plugin $=1$.

Escardibul, Josep Oriol, Morales, Susana, Pérez, Carmen y De la Torre, Eva (2013): "Evolución de los precios de matrícula en las enseñanzas universitarias por CCAA (1992-2013): un análisis antes después del Espacio Europeo de Educación Superior”. Comunicación en Jornadas de la Asociación de Economía de la Educación (AEDE), Asociación de Economía de la Educación (AEDE), Universidad de La Coruña.

Gil, Fernando. (2002). La exclusión social. Barcelona: Editorial Ariel.

Hernández Armenteros Juan y Pérez García, José A. (2013): Informe sobre el “Proyecto de Real Decreto por el que se establecen los umbrales de renta y patrimonio familiar y las cuantías de las becas y ayudas al estudio para el curso 2013- 2014". http://www.comunicacion.us.es/sites/default/files/Documento $\% 20$ de $\% 20$ trabajo $\% 20 \mathrm{RD} \% 20$ Becas.pdf, consultado el 25 de noviembre de 2016.

Hernández Pedreño, Manuel (2010): "El estudio de la pobreza y la exclusión social. Aproximación cuantitativa y cualitativa". Revista Interuniversitaria de Formación del Profesorado, 24, 25-46. http://www.redalyc. org/articulo.oa?id=27419173003, consultado el 15 de noviembre de 2016. 
IDESCAT (2012). Població en risc de pobresa o d'exclusió social. Disponible en: http:/ /www.idescat.cat/ economia $/$ inec?tc $=3 \&$ id $=8510$.

INE (2014). Encuesta de Población Activa del primer trimestre de 2014. Disponible en: http://www.ine. es/inebaseDYN/epa30308/epa_inicio.htm.

Langa Rosado, Delia y Río Ruiz, Manuel Ángel (2013): “Los estudiantes de clases populares en la universidad y frente a la universidad de la crisis: persistencia y nuevas condiciones para la multiplicación de la desigualdad de oportunidades educativas". Tempora. Revista de Sociología de la Educación, 16, 71-96.

Laparra, Miguel. (2010). Elprimer impacto de la crisis en la cohesión social en España. Madrid: Fundación Foessa).

Ley 3/2012, de 6 de julio, de medidas urgentes para la reforma del mercado laboral. (BOE núm. 162, 7 de julio de 2012).

Ley Orgánica 15/1999, de 13 de diciembre, de Protección de Datos de Carácter Personal. (BOE núm. 298, 14 de diciembre de 1999).

Ley Orgánica 8/2013, de 9 de diciembre, para la mejora de la calidad educativa. (BOE núm. 295, 10 de diciembre de 2013).

Lorenzo, Francisco. (Dir.). (2014). VII Informe sobre exclusión y desarrollo social en España. (Madrid. Fundación Foessa).

Navarro, José (2014): "La inserción laboral de los titulados universitarios de clase obrera en Cataluña". Revista de la Asociación de Sociología de la Educación, 7 (2), 488-510.

Observatori de l'Estudiant de la Univeritat de Barcelona (2016). Informe executiu. Enquesta sobre condicions de vida i participación dels estudiants universitaris ECoVIPEU-UB. Disponible en http://hdl. handle.net/2445/104204.

Organización de las Naciones Unidas (1995). The Copenhagen Declaration and Programme of Action. Disponible en: http://www.unesco.org/education/pdf/COPENHAG.PDF.

Organización de las Naciones Unidas (2015). World Economic Situation and Prospect 2015. Disponible en: http://www.un.org/en/development/desa/policy/wesp/

Unión General de Trabajadores (2014). Boletín Precariedad Popular. Disponible en: http://www.ugt.es/ Publicaciones/bolet\%C3\%ADn-prepariedad-popular-JUVENTUD.pdf.

Unión General de Trabajadores (2014). Dos años de reforma laboral: evolución del empleo, la contratación, los despidos y la negociación colectiva. Disponible en; http://www.ugtextremadura.org/userfiles /029667153f3d6854fa13d283a74689e3.pdf.

Real Decreto 1000/2012, de 29 de junio, por el que se establecen los umbrales de renta y patrimonio familiar y las cuantías de las becas y ayudas al estudio, para el curso 2012-2013 y se modifica parcialmente el Real Decreto 1721/2007, de 21 de diciembre, por el que se establece el régimen de las becas y ayudas al estudio personalizadas. (BOE núm. 160, 5 de julio de 2012).

Real Decreto 609/2013, 2 de agosto, por el que se establecen umbrales de renta y patrimonio familiar y las cuantías de las becas y ayudas al estudio para el curso 2013-2014, y se modifica particularmente el real decreto $1721 / 2007$, de 21 de diciembre, por el que se establece el régimen de las becas y ayudas al estudio personalizado. (BOE núm. 185, 3 de agosto de 2013). 
Débora García Barrios, Jorge Luis Méndez-Ulrich. Estudiantes universitarios en riesgo... RASE 2017. Vol. 10. N. ${ }^{\circ}$ 1: Págs. 37-49 doi: http://dx.doi.org/10.7203/RASE. 10.1.8996

Río Ruiz, Manuel Ángel. (2014): “Efectos de la conversión en becario y consecuencias de la reforma del sistema de becas entre universitarios de clase obrera". Revista de la Asociación de Sociología de la Educación, 7 (2), 468-487.

Subirats, Joan (dir.) (2004): Pobreza y exclusión social. Un análisis de la realidad española y europea. Barcelona: Fundación La Caixa. 